\title{
REGIONAL ANNULUS FIBRE ORIENTATIONS USED AS A TOOL FOR THE CALIBRATION OF LUMBAR INTERVERTEBRAL DISC FINITE ELEMENT MODELS
}

\author{
A. Malandrino ${ }^{1}$, J. Noailly ${ }^{2}$ and D. Lacroix ${ }^{3}$
}

\section{ABSTRACT}

The highly organized collagen network of human lumbar annulus fibrosus (AF) is fundamental to preserve the mechanical integrity of the intervertebral discs. In the healthy AF, fibres are embedded in a hydrated matrix and arranged in a criss-cross fashion, giving an anisotropic structure capable to undergo large strains. Quantitative anatomical examinations revealed particular fibre orientation patterns, possibly coming from regional adaptations of the AF mechanics. Based on such hypothesis, this study aimed to show that the regional differences in AF mechanical behaviour can be reproduced by considering only fibre orientation changes. Using the finite element (FE) method, AF matrix was modelled as a poro-hyperelastic material, where the porous solid was treated as a compressible continuum following a Neo-Hookean constitutive law. Strain-dependent permeability was assumed and all material parameters were taken from the literature. Fibre reinforcement was accounted for by adding an extra-term to the porous matrix strain energy density function, only active along the fibre directions. Through such term, fibre orientations were then adjusted, to reproduce AF tensile behaviours measured for four different regions: posterior outer (PO), anterior outer (AO), posterior inner (PI) and anterior inner (AI). Curve calibrations resulted in the following optimal angles, calculated with respect to the circumferential axis: $28^{\circ}$ for PO, $23^{\circ}$ for $\mathrm{AO}, 43^{\circ}$ for PI and $31^{\circ}$ for $\mathrm{AI}$. In average, we obtained fibres $30 \%$ more transversal in the inner than in the outer AF against 38\% as measured by Cassidy et al. (1989). Fibres more axial in the posterior than in the anterior AF were also measured by Holzapfel et al. (2005), with angle values comparable to our computed average values. Since all the hyperelastic and fluid-phase material parameters remained unchanged throughout the AF, calibration based only on fibre patterns variations may be an effective tool to calibrate the regional AF mechanics in a realistic way.

\section{INTRODUCTION}

A challenge for FE models of the intervertebral disc (IVD) AF is to capture its intricate structure and function. The concentric fibre-reinforced lamellae that build up the AF are strongly anisotropic, due to collagen fibre orientation patterns. At the same time the annulus presents significant regional differences in mechanical behaviour. For instance, under a moderate strain of 5-6\%, specimens in the AO region of the AF experienced stresses about 10 times higher than those occurring in PI specimens ${ }^{1}$. Collagen fibres, embedded in a criss-cross pattern within the AF matrix are essential to properly

\footnotetext{
${ }^{1}$ MSc, Biomechanics and Mechanobiology Research Group, Institute for Bioengineering of Catalonia (IBEC), Baldiri Reixac, 4, Barcelona 08028, Spain

${ }^{2} \mathrm{PhD}$, Biomechanics and Mechanobiology Research Group, IBEC

${ }^{3} \mathrm{PhD}$ and Group Leader, Biomechanics and Mechanobiology Research Group, IBEC
} 
mechanically stabilize the IVD and tune the functional biomechanics of the intersegmental joints. Interestingly, regional variations in AF fibre criss-cross angles have been anatomically observed ${ }^{2,3,4}$, which could be related consistently with the regional-dependent functional biomechanics of the AF tissue ${ }^{5}$.

As a result of the several fibre angle distributions in the literature, different fibre patterns could be used as input for models. For example, following the description from Marchand and Ahmed ${ }^{6}$, some studies did not model any particular changes of fibre angle throughout the AF, taking an average criss-cross angle value of $+/-30^{\circ}$ with respect to the AF circumferential direction ${ }^{7}$. According to Cassidy et al. ${ }^{4}$ other authors introduced in their model more transversal fibres in the inner than in the outer $\mathrm{AF}^{8}$. Another type of fibre-induced AF anisotropy model ${ }^{2,9}$ was based on the observations of Holzapfel et al. ${ }^{2}$ who reported an increasing fibre angle with respect to the AF transverse plane, in the tangential direction, from the anterior to the posterior annulus. More recently, Zhu et al. ${ }^{3}$ investigated the lumbo-sacral annulus microstructure and reported both radial and tangential AF criss-cross angle changes. However, to our knowledge, this later configuration has not been modelled so far.

In FE models of the AF, the collagen network is implemented by using fibre-reinforced elements $^{7,8}$, as well as through continuum constitutive theories that can take into account fibre orientations and fibre stiffness-related parameters ${ }^{2,9}$. In particular, by using a continuum approach, Eberlein and coworkers ${ }^{9}$ showed how varying the fibre stiffness-related parameters, two different mechanical behaviours for posterior and anterior AF regions can be reproduced. They implemented as well such AF constitutive behaviour in a FE model of the whole lumbar IVD, to study the functional disc behaviour. The regional variations in fibre angles were studied only circumferentially and the experimental stress-strain responses were calibrated by varying the fibre stiffness parameters. However in their study a full validation could not be obtained. Moreover, anatomical data on radial gradients in AF collagen network could be important to stabilize the motion segments ${ }^{7}$ while explaining the differences in tissue mechanical response between inner and outer specimens ${ }^{5}$.

In the present study, we propose a novel model calibration procedure where we treat the fibre angles as unknown parameters, under the hypotheses of non variability for the mechanical properties of the AF matrix. For this, a continuum anisotropic porohyperelastic approach was chosen and regional AF stress-strain behaviours were reproduced by locally calibrating fibre orientation and stiffness based on reported mechanical tests on different AF samples. Calibration results in terms of fibre angles were finally implemented in a complete FE IVD model to integrate the locally calibrated fibre patterns in the global context of IVD biomechanics.

\section{METHODS}

To define the mechanical behaviour of the AF solid phase, an anisotropic hyperelastic continuum approach was chosen. The total strain energy density function $W_{A F}$ presents an extra term $W_{F}$ in which the strain-like quantity $\bar{E}_{\alpha}$ is active only in two opposite orientations ${ }^{9}$, reproducing the fibre criss-cross pattern:

$$
W_{A F}=W_{M}+W_{F}=\frac{G}{2}\left(I_{1}-3\right)+\frac{K}{2}(J-1)^{2}+\frac{K_{1}}{K_{2}} \sum_{\alpha=1}^{2}\left\{\exp \left[K_{2}\left\langle\bar{E}_{\alpha}\right\rangle^{2}\right]-1\right\}
$$


where $G$ and $K$ are respectively the shear and bulk modulus, $K_{1}$ and $K_{2}$ are fibre stiffness-related parameters, $I_{1}$ is the first strain invariant and $W_{M}$ represents the strain energy density of the fibre-embedding porous matrix, for which a compressible NeoHookean model was used. Strain dependence for permeability $k$ was implemented following an empirical law ${ }^{7}$, in which permeability is a function of the current void ratio $e$, its initial value $e_{0}$, initial permeability $k_{0}$, and an empirical coefficient $M$ :

$$
k=k_{0}\left[\frac{e\left(1+e_{0}\right)}{e_{0}(1+e)}\right]^{2} \exp \left[M\left(\frac{1+e}{1+e_{0}}-1\right)\right]
$$

In such a poro-hyperelastic view, the total stress $\underline{\underline{\sigma}}$ caused by external loading and consequent fluid velocity $\dot{u}_{f}$ are given by

$$
\begin{gathered}
\underline{\underline{\sigma}=\frac{1}{J}} \frac{\partial W_{A F}}{\partial \underline{\underline{F}}} \underline{F}^{T}-\frac{e}{1+e} p \underline{\underline{I}} \\
\dot{u}_{f}=-k \nabla p
\end{gathered}
$$

being $\underline{\underline{F}}$ the deformation gradient tensor, $J$ the volumetric strain, and $p$ the interstitial pore pressure. $G$ and $K$ were calculated from experimentally measured biphasic properties $^{10}$ (assuming a Poisson's ratio $v=0.2^{11}$ ). $K_{1}$ and $K_{2}$ for the anterior and posterior AF region were chosen from literature by hypothesizing only circumferential stiffness changes ${ }^{9}$. In the present study, a further radial subdivision was assumed for the calibration of fibre angles (Fig. 1). Table 1 summarizes all values chosen.

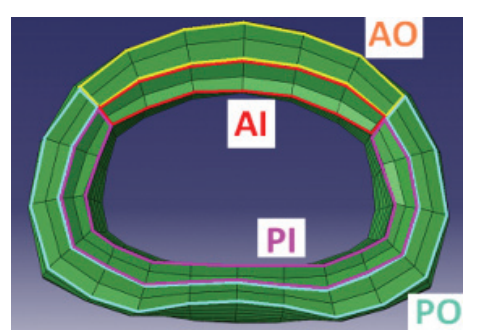

Figure 1: Regional subdivision for the annulus fibrosus. First letter refers to circumferential ( $\mathrm{A}=$ anterior, $\mathrm{P}=$ posterior) whereas second refers to the radial one $(\mathrm{O}=$ outer, $\mathrm{I}=$ inner $)$.

\begin{tabular}{|c|c|c|c|c|c|c|}
\hline$G(\mathrm{MPa})$ & $K\left(\mathrm{MPa}^{-1}\right)$ & $k_{0}\left(\mathrm{~m}^{4} \mathrm{~N}^{-1} \mathrm{~s}^{-1}\right)$ & $e_{0}$ & $M$ & $K_{l}(\mathrm{MPa})$ & $K_{2}$ \\
\hline 0.28 & 0.37 & $0.2 \times 10^{-15}$ & 2.33 & \multirow{2}{*}{1.18} & Anterior 2 & Anterior 190 \\
\cline { 5 - 7 } & & & & Posterior 5 & Posterior 10 \\
\hline
\end{tabular}

Table 1: Material properties for the Neo-Hookean strain energy function, strain dependent permeability (eq. (2)) and fibre related material properties.

An experimental set-up ${ }^{1}$ on AF tissue samples (Fig 2) was reproduced through a FE analysis and stress-strain experimental curves were calibrated by varying only the fibre angles, for the four regions considered: AF, AO, PI and PO.

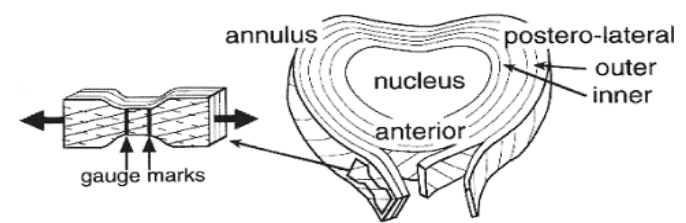

Figure 2: tissue sample experiment simulated from [1]. 
Additionally, a $500 \mathrm{~N}$ compressive creep experiment on cadaveric L4-L5 discs ${ }^{12,13}$ was simulated to evaluate both the global IVD mechanical response and the fibre strain values obtained with the calibrated fibre angles. The undeformed local fibre orientations were defined with respect to the local element orientation system that followed the radial and tangential directions the AF geometry (Fig. 1). A large strain formulation was chosen, so that, fibre directions were automatically updated at each load increment, according to the current deformation.

Nucleus pulposus was considered as poro-hyperelastic with a Neo Hookean strain energy function, but not fibre-reinforced, due to random orientation of collagen fibres observed in NP tissues. Cartilage and bony endplates were treated as poroelastic (Table 2). Calculations were done with the commercial software ABAQUS.

\begin{tabular}{|c|c|c|c|c|l|l|}
\hline & $G(\mathrm{MPa})$ & $K\left(\mathrm{MPa}^{-1}\right)$ & $k_{0}\left(\mathrm{~m}^{4} \mathrm{~N}^{-1} \mathrm{~s}^{1}\right)$ & $e_{0}$ & References & Notes \\
\hline $\begin{array}{c}\text { Nucleus } \\
\text { Pulposus }\end{array}$ & 0.1125 & 0.15 & $9 \times 10^{-16}$ & 4.88 & $\begin{array}{l}\text { Perie et al.., 2004 } \\
\text { Ferguson et al., 2004 }\end{array}$ & $\begin{array}{l}\text { Poro-hyperelastic, strain- } \\
\text { dependent permability } \\
\text { (eq. (2), M=8.5) }\end{array}$ \\
\hline $\begin{array}{c}\text { Cartilage } \\
\text { endplate }\end{array}$ & 7.14 & 33.3 & $2.52 \times 10^{-15}$ & 4 & $\begin{array}{l}\text { Almeida and Spilker } \\
1997^{14} ; \text { Ferguson et al., } \\
2004^{11}\end{array}$ & $\begin{array}{l}\text { Poroelastic, strain } \\
\text { dependent permeability } \\
\text { (eq. (2), M=4.6) }\end{array}$ \\
\hline $\begin{array}{c}\text { Bony } \\
\text { endplate }\end{array}$ & 384.6 & 833.3 & $2.68 \times 10^{-8}$ & 0.05 & $\begin{array}{l}\text { Whyne et al., 2001 } \\
\text { Ferguson et al, 2004 }\end{array}$ & $\begin{array}{l}\text { Poroelastic, constant } \\
\text { permeability } \mathrm{k}_{0}\end{array}$ \\
\hline
\end{tabular}

Table 2: Material properties chosen for NP, cartilage and bony endplates. For endplates, $G$ and $K$ were calculated from reported Young's modulus and Poisson's ratio.

\section{RESULTS}

Curve calibrations (Fig. 3) resulted in the following optimal angles, calculated with respect to the circumferential axis: $28^{\circ}$ for $\mathrm{PO}, 23^{\circ}$ for $\mathrm{AO}, 43^{\circ}$ for $\mathrm{PI}$ and $31^{\circ}$ for $\mathrm{AI}$. Fig. 4 reports the values averaged so that they can be compared with the anatomical studies that found only radial ${ }^{4}$ and circumferential ${ }^{2}$ variations, respectively.

\section{Calibration}

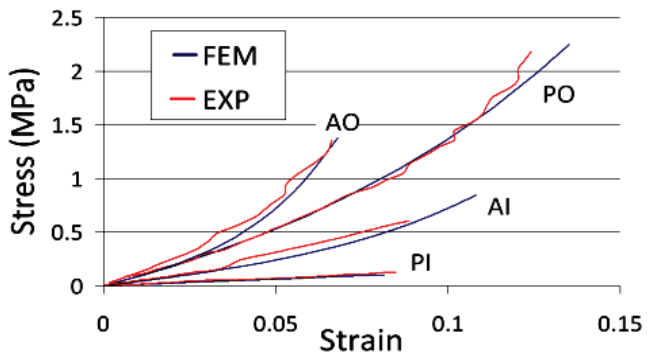

Figure 3: Calibration of stress-strain curves with experimental data ${ }^{3}$.
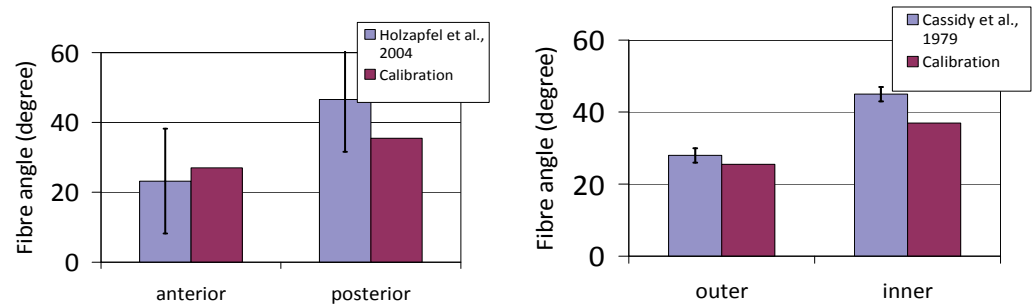

Figure 4: Averaged data for regional variations in fibre angles resulting from calibration, comparison with the two patterns ( radial $^{4}$ and circumferential ${ }^{2}$ ).

Fig. 5 shows the creep predictions compared with literature experimental data ${ }^{12}$, in terms of NP intradiscal pressure and disc height changes. With the simulated 
compressive force $(500 \mathrm{~N})$, maximum surface strains in the collagen fibre direction were $6 \%$ in the postero-lateral region of the $\mathrm{AF}$, against $6.7 \%$ reported in literature ${ }^{13}$
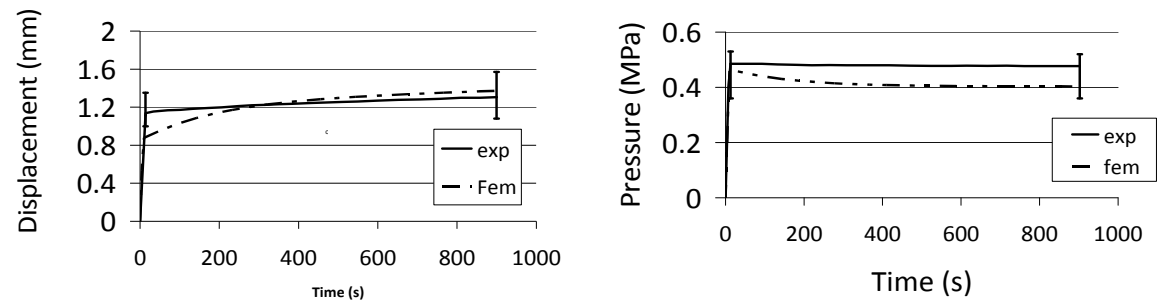

Figure 5: FE model results with AF regional properties derived from the regional fibre angle differences in terms of disc height reduction (left) and NP intradiscal pressure (right) and comparison with experimental values ${ }^{12}$.

\section{DISCUSSION}

Results demonstrated that, while maintaining the embedding matrix properties constant throughout the AF, it is possible to follow the AF regional mechanics by regional calibration of fibre orientations only. The results in terms of fibre angles are in general in agreement with the collagen fibre regional patterns found in the literature ${ }^{2,4}$. Results also show that a radial and tangential variations in fibre angle based on experiments at the tissue level produce a satisfactory response when implemented in a whole FE disc model. Moreover, in a complete lumbar spine functional unit, the tangential fibre pattern is thought to be adapted to the mechanical action of the facet joints (posterior) ${ }^{5}$. Radial changes could be explained considering a better mechanical response when intersegmental shear deformations are involved (e.g. in axial rotation loading modes).

It is worth to highlight that the values found for fibre angles depended on the embedding matrix material properties. Since experimental variability and swelling pretesting conditions affect these properties ${ }^{10}$, calibration results are dependent from the experiments or parameter values chosen in the literature for the model definition. However, the present study clearly shows that the calibration procedure, based on regional variability for global tensile AF mechanical behaviour, is a valid tool once matrix material parameters have been carefully determined, since it produces fibre patterns observed in anatomical measurements.

In the present model, four fibre angle discrete regions were considered, whereas smooth gradients (i.e. a linear pattern for fibre variation) have been measured in both radial and tangential directions ${ }^{2,3,4}$. Future work should include a less abrupt fibre angle changes from one region to another, by including those gradients for fibre angles throughout the AF. Differences in AF regional mechanics could also be due to regional variation of AF matrix properties ${ }^{10}$. However, our calibration approach is based on the simpler approach of considering all the anisotropy due to the fibres rather than the matrix.

In conclusion, this study shows that fibre angles are able to reproduce the regional AF tissue mechanics and lead to a satisfactory global mechanical behaviour when implemented in a complete IVD FE model. Therefore, calibration based only on fibre patterns and stiffness variations may be a simple and effective tool to reproduce the AF biomechanical behaviour in a realistic way.

\section{ACKNOWLEDGEMENTS}

Funding was obtained from the European Commission (DISC REGENERATION- 
NMP3-LA-2008-213904) and from the Spanish Ministry of Science and Innovation (FPU program) through the pre-doctoral fellowship (AP2008-03317)

\section{REFERENCES}

1. Ebara S., Iatridis J. C., Setton L. A., et al. Tensile properties of nondegenerate human lumbar anulus fibrosus, Spine, 1996, 21, 452-461.

2. Holzapfel G. A., Schulze-Bauer C. A. J., et al. Single lamellar mechanics of the human lumbar anulus fibrosus, Biomechan. Model. Mechanobiol., 2005, 3, 125-140.

3. Zhu D., Gu G.S., et al. Micro-structure and mechanical properties of annulus fibrous of the L4-5 and L5-S1 intervertebral discs, Clin. Biomech., 2008, 23, S74-82.

4. Cassidy J.J., Hiltner A. et al Hierarchical structure of the intervertebral-disk, Conn. Tiss. Res., 1989, 23, 75-88.

5. Noailly, J. and Lacroix, D. (2010) Significance of the collagen criss-cross angle distributions in lumbar annuli fibrosi as revealed by finite element simulations. Proceedings of the 9th International Symposium on Computer Methods in Biomechanics and Biomedical Engineering, Valencia, Spain, March 24-27. In press.

6. Marchand F. and Ahmed A.M. Investigation of the laminate structure of lumbar disc anulus fibrosus, Spine, 1990, 15, 402-410.

7. Argoubi M. and Shirazi-Adl, A., Poroelastic creep response analysis of a lumbar motion segment in compression. J. Biomech., 1996, 29, 1331-1339.

8. Noailly J., Wilke H.-J., et al. How does the geometry affect the internal biomechanics of a lumbar spine bi-segment finite element model? Consequences on the validation process. J. Biomech., 2007, 40, 2414-2425.

9. Eberlein, R., Holzapfel G.A. et al., An anisotropic model for annulus tissue and enhanced finite element analyses of intact lumbar disc bodies. Comput. Methods Biomech. Biomed. Engin., 2001, 209-230.

10. Perie D., Korda D. et al. Confined compression experiments on bovine nucleus pulposus and annulus fibrosus: sensitivity of the experiment in the determination of compressive modulus and hydraulic permeability. J. Biomech., 2005, 38, 2164-71.

11. Ferguson S. J., Ito K. and Nolte P., Fluid flow and convective transport of solutes within the intervertebral disc, J. Biomech., 2004, Vol 37, 213-221.

12. Heuer F., Schmitt H., Schmidt H., Claes L. and Wilke H., Creep associated changes in intervertebral disc bulging obtained with a laser scanning device, Clin. Biomech., 2004, Vol. 22, 737-744.

13. Heuer F., Schmidt H., et al. The relation between intervertebral disc bulging and annular fiber associated strains for simple and complex loading J. Biomech., 2008, 1086-1094.

14. Almeida E.S. and Spilker R.L. Mixed and Penalty Finite Element Models for the Nonlinear Behavior of Biphasic Soft Tissues in Finite Deformation: Part II Nonlinear Examples. Comput. Methods Biomech. Biomed. Engin., 1997, 1, 151-70.

15. Whyne C.M., Hu S.S., et al. Parametric finite element analysis of vertebral bodies affected by tumors, J. Biomech., 2001, 34, 1317-1324. 